\title{
DNA Hydroxymethylation
}

National Cancer Institute

\section{Source}

National Cancer Institute. DNA Hydroxymethylation. NCI Thesaurus. Code C106316.

A DNA modification process where a hydrogen at the C5-position of a cytosine residue is replaced with a hydroxmethyl group. 